\title{
Video-assisted thoracic surgery for tiny pulmonary nodules with real-time image guidance in the hybrid theatre: the initial experience
}

\author{
Peter S. Y. Yu ${ }^{1}$, Cheuk Man Chu ${ }^{2}$, Rainbow W. H. Lau ${ }^{1}$, Innes Y. P. Wan ${ }^{1}$, Malcolm J. Underwood ${ }^{1}$, Simon \\ C. H. Yu ${ }^{2}$, Calvin S. H. $\mathbf{N g}^{1}$ \\ ${ }^{1}$ Division of Cardiothoracic Surgery, Department of Surgery, ${ }^{2}$ Department of Imaging and Interventional Radiology, The Chinese University of \\ Hong Kong, Prince of Wales Hospital, Hong Kong, China \\ Contributions: (I) Conception and design: CS Ng, SC Yu, PS Yu; (II) Administrative support: CS Ng, IY Wan, SC Yu, MJ Underwood; (III) Provision \\ of study materials or patients: CM Chu, RW Lau, IY Wan, SC Yu, MJ Underwood, CS Ng; (IV) Collection and assembly of data: PS Yu; (V) Data \\ analysis and interpretation: PS Yu; (VI) Manuscript writing: All authors; (VII) Final approval of manuscript: All authors. \\ Correspondence to: Calvin S. H. Ng, MD, FRCS, FCCP. Division of Cardiothoracic Surgery, Department of Surgery, The Chinese University of Hong \\ Kong, Prince of Wales Hospital, 30-32 Ngan Shing Street, Shatin, New Territories, Hong Kong, China. \\ Email: calvinng@surgery.cuhk.edu.hk.
}

\begin{abstract}
Background: Localization of tiny lung nodules during video-assisted thoracic surgery (VATS) resection can be challenging. Real-time image-guided hookwire localization of the target lesions immediately followed by VATS lung resection in the hybrid operating theatre setting is an emerging approach.

Methods: We retrospectively reviewed our experience with this form of hybrid operating theatre imageguided VATS (iVATS) for lung nodules $1.5 \mathrm{~cm}$ or less, or soft in consistency. These patients were compared with matched cohort who received standard hookwire localization in the radiology department.

Results: From February 2014 to September 2017, lung nodules of indeterminate nature in 32 consecutive patients with mean size $9.1 \pm 4.6 \mathrm{~mm}$ underwent iVATS. All were accurately localized by hookwire and successfully resected. There was no postoperative mortality. There were 21 (66\%) malignant lesions, all with adequate resection margins. Major outcomes were compared with a comparable cohort of 8 patients who received standard hookwire localization and VATS (sVATS) performed at separate departments operation suites. sVATS groups has significantly longer 'at-risk' period for pneumothorax progression and hookwire dislodgement $(109.5 \pm 57.1$ minutes for sVATS $v s$. 41.1 \pm 15.0 minutes for iVATS, $\mathrm{P}=0.011)$, and a higher risk of hookwire dislodgement ( $25 \%$ for sVATS $v$ s. 0 for iVATS, $\mathrm{P}=0.036$ ).

Conclusions: Real-time image-guided hookwire localization in the hybrid theatre setting is an effective facilitator of VATS resection of tiny lung nodules in selected patients, and may have added advantages in terms of safety and localization accuracy over the conventional sVATS method.
\end{abstract}

Keywords: Pulmonary nodule; localization; hybrid theatre; video-assisted thoracic surgery (VATS)

Submitted Mar 04, 2018. Accepted for publication May 03, 2018.

doi: $10.21037 /$ jtd.2018.05.53

View this article at: http://dx.doi.org/10.21037/jtd.2018.05.53

\section{Introduction}

Localization is an important step in wedge resection of lung nodules with a view to accurate resection and achieving adequate surgical margin. For lesions that are small, soft in consistency (e.g., with high ground-glass opacity percentage), or distant from the visceral pleura (e.g., depth greater than twice lesion diameter) (1), digital palpation as the simplest way of intraoperative localization can be unreliable. Furthermore, the limited access during minimally invasive thoracic surgery can increase the difficulty of nodule localization. In the appropriate clinical 
context, such as history of pulmonary or extra-pulmonary malignancy in which pulmonary recurrence or metastasis remain a top differential diagnosis, continuing imaging surveillance of these small nodules may delay diagnosis and treatment of the condition. In certain cases surgical resection can be both diagnostic and therapeutic.

Various adjuncts to allow proper localization of such nodules have been described, with hookwire insertion under radiological guidance being one of the most popular modalities. Currently, most of the localization procedures are performed in the radiology suite under image guidance. However, often there is a significant period of delay between hookwire insertion in the radiology suite and the lung surgery. This can pose some safety concerns. First, bleeding and pneumothorax are known complications from hookwire insertion, therefore close monitoring and specialist nursing care is required following the procedure. Occasionally, additional interventions, such as chest drain insertion, are required during the waiting period prior to surgery. Secondly, multiple inter-departmental transfer and patient movements increase the risk of migration or even dislodgement of hookwires, resulting in failure of proper localization of lung nodules.

By performing the localization procedures in the same suite as the lung operation in the hybrid operation theatre setting, these potential risks could be minimized (2). We report our early experience of using real-time imageguided hookwire localization of small pulmonary nodules immediately followed by video-assisted thoracic surgery (iVATS) for pulmonary wedge resection. Furthermore, patients who underwent iVATS were compared with matched cohort who received standard hookwire localization (sVATS) in the radiology department.

\section{Methods}

\section{The hybrid theatre}

Our hybrid operating theatre is a $10 \mathrm{~m} \times 7 \mathrm{~m}$ room with a robot-supported, fixed three-dimensional imaging system (Artis zeego system, Siemens Healthcare, Erlangen, Germany). Multiple LCD monitors inside the OR provides real-time imaging feedbacks. A side control room is equipped with a radiology console (syngo $\mathrm{X}$ Workplace, Siemens Healthcare, Erlangen, Germany) capable of performing calculations, measurements, and complex three-dimensional image reconstructions. The operating room also has the standard facilities and equipment to carry out complex minimally invasive video-assisted thoracic surgery (3).

\section{Image-guided VATS (iVATS) wedge resection}

Depending on the location of the target lung nodule, patient is positioned appropriately in the supine, lateral decubitus or prone position for hookwire insertion. On the control console, the interventional radiologist determines the optimal direction and depth of needle puncture in multiple view planes based on the cone-beam CT image (syngo ${ }^{\circledR}$ DynaCT, Siemens Healthcare, Erlangen, Germany). The hookwire (SOMATEX ${ }^{\circledR}$ Medical Technologies $\mathrm{GmbH}$, Germany) is then deployed and fixed. Patient is immediately repositioned, undergo general anesthesia with double-lumen endotracheal tube, and receive VATS wedge resection performed in the usual manner (Figure 1). All the iVATS hookwire insertions were performed by the interventional radiologist, in the presence of the operating surgeon to provide guidance to avoid wire placement that may negatively affect VATS. The wedge resection of the target lesion is guided by: (I) the direction of the hookwire; (II) the distance from the tip of the hookwire in relation to the target lesion as measured by the real-time DynaCT image (Figure 2); and (III) the puncture mark by the hookwire on visceral pleura surface with the distance of the lesion measured by the DynaCT image. Should there be any uncertainty about the accuracy of hookwire positioning during the operation, on-table CT imaging can be performed to confirm the accuracy of localization and to reinsert the hookwire if necessary. The hookwire may be cut short within the pleural cavity, or removed before the wedge resection, depending on the ease of lung manipulation and staple line. The specimen is delivered together enclosed inside a specimen bag. A completion lobectomy will follow if the intraoperative frozen section confirmed lung primary malignancy.

\section{Patient cohort}

We have started performing iVATS wedge resection of small pulmonary nodules in the hybrid OR since February 2014. iVATS was performed for: any lesion with size not bigger than $1.5 \mathrm{~cm}$ or ground-glass opacity (GGO) with or without solid component. iVATS was not performed if the lung nodules were inaccessible by percutaneous approach, for example, those lesions that are shielded by the scapula and clavicle, or are at proximity to visceral 


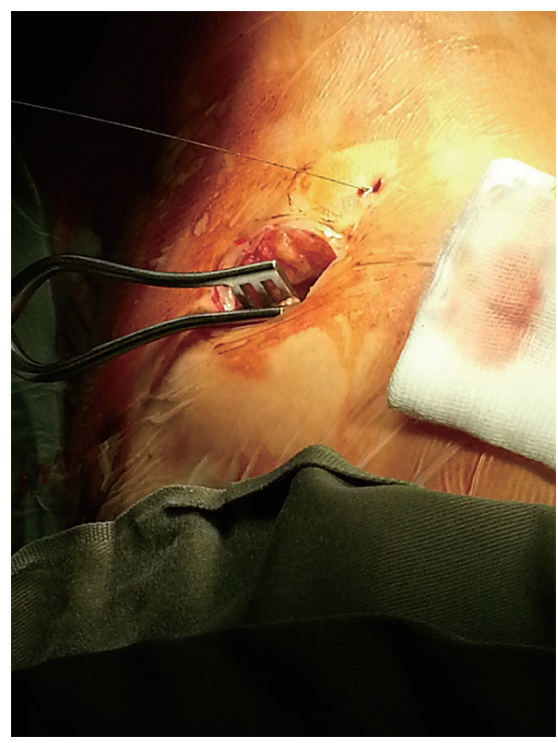

Figure 1 Intraoperative photo showing the creation of single port for video-assisted thoracic surgery (VATS) for a small pulmonary nodule localized by hookwire in the same hybrid theatre setting.

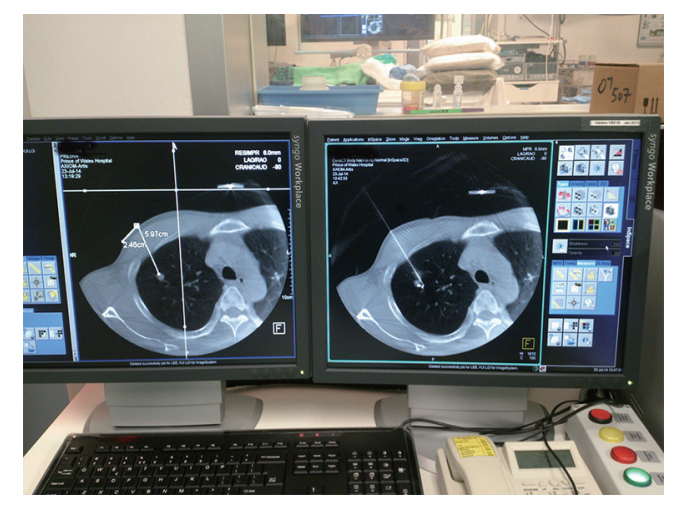

Figure 2 Hybrid operating room DynaCT reconstructed images showing (left) the planning of percutaneous hookwire approach, and (right) following hookwire insertion with placement immediately adjacent to the lesion.

organs and major neurovascular structures. The 'at-risk' period, during which patients were at risk of developing progressive pneumothorax and hookwire dislodgement, was defined as the period between the completion of hookwire insertion and the commencement of VATS. Prior to the availability of the hybrid theatre, patients had separate procedure and operation suites for hookwire localization and VATS (sVATS), and this group of patients was selected as the control group. The sVATS cohort is limited because before the availability of hybrid theatre, there was a general tendency to manage small lesions with serial scans until they were more easily localizable during surgery. The primary outcomes of measure are the accuracy of localization, the ability to achieve clear resection margins, and the duration of 'at-risk' period. Secondary outcomes, such as pneumothorax, bleeding, hookwire malposition, migration or dislodgement, and post-operative outcomes (e.g., mortality, complications, chest drain duration, length of stay) were also assessed. Hookwire dislodgement is defined as the hookwire being completely dislodged from and with no identifiable marking on the visceral pleura surface.

The ethical approval is by the Joint Chinese University of Hong Kong-New Territories East Cluster Clinical Research Ethics Committee (CREC Ref No. 2017-292). Patient consent for the study is not necessary in view of its retrospective nature.

\section{Statistical analysis}

Descriptive statistics were used to compare the variable between the groups. Continuous variables following Normal distribution were reported as mean ( \pm standard deviation) and compared by two-sample (unpaired) $t$-test. Continuous variables not following a Normal distribution were reported as median value (+ inter-quartile range) and compared using the Mann-Whitney test. Categorical variables were reported as counts and percentages, and differences between two groups were assessed by Chi-square test, or Fisher's exact test if a cell value was lower than 5 . All statistics were analysed using the SPSS (Version 24.0, IBM, Armonk, NY, USA). A $\mathrm{P}<0.05$ represents statistically significant difference.

\section{Results}

From February 2014 to September 2017, 32 consecutive patients received iVATS in the hybrid OR. The demographic and operative characteristics are summarized in Tables 1,2. On computer tomography scan, the lung nodules of all the patients were small (mean size $9.1 \pm 4.6 \mathrm{~mm}$ ), most were soft or of ground-glass consistency (66\%), and distant from the visceral pleura (shortest distance from visceral pleura to nodule: $10.8 \pm 8.3 \mathrm{~mm}$; depth-to-diameter ratio $1.5 \pm 1.5$ )

Pneumothorax was detected in 15 (47\%) iVATS patients on real-time CT scan immediately after hookwire placement. As all patients will immediately proceed to 
Table 1 Demographics of the iVATS vs. sVATS cohort

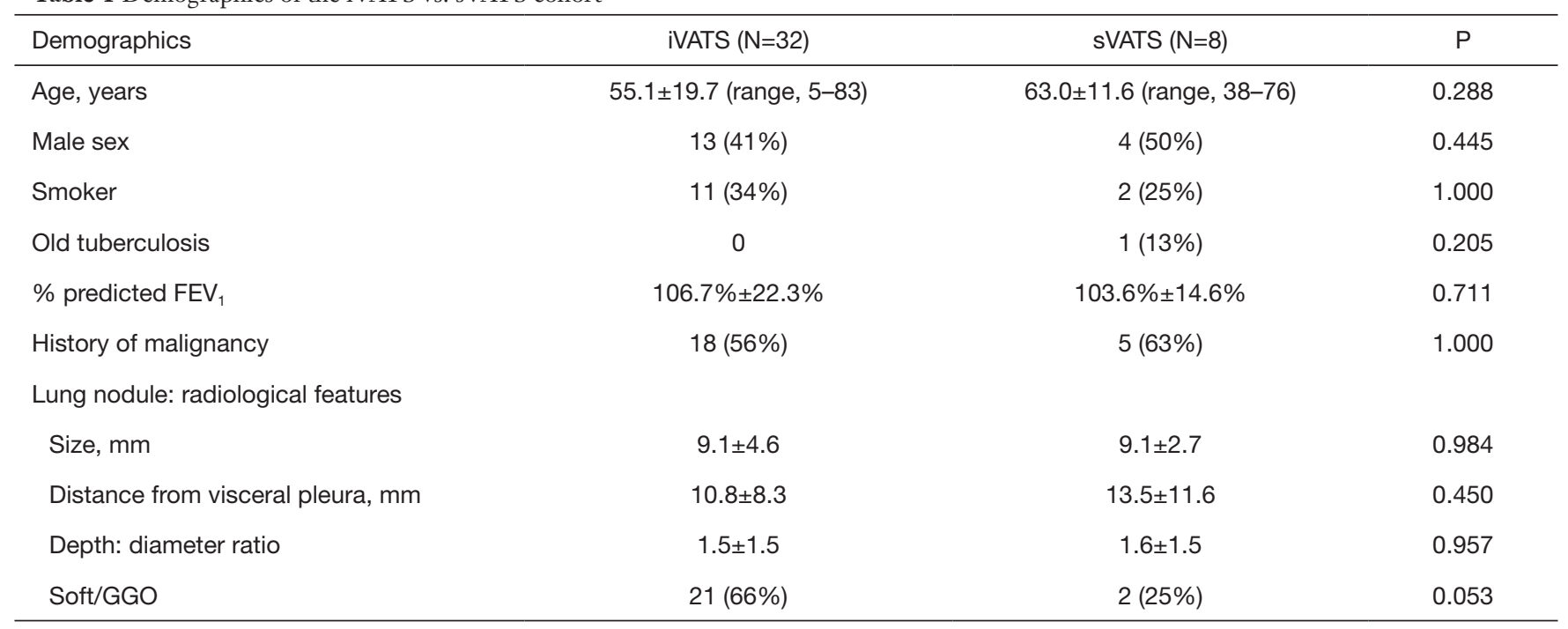

$\mathrm{FEV}_{1}$, forced expiratory volume in the first second; GGO, ground glass opacity.

Table 2 Operative details and outcomes of the iVATS vs. sVATS cohort

\begin{tabular}{|c|c|c|c|}
\hline Operative parameters & iVATS (N=32) & sVATS $(\mathrm{N}=8)$ & $\mathrm{P}$ \\
\hline Pneumothorax after hookwire insertion & $15(47 \%)$ & $6(75 \%)$ & 0.154 \\
\hline Duration of 'at-risk' period (minutes) & $41.1 \pm 15.0$ & $109.5 \pm 57.1$ & 0.011 \\
\hline Dislodged hookwire & 0 & $2(25 \%)$ & 0.036 \\
\hline Upper lobe procedure & $8(25 \%)$ & $4(50 \%)$ & 0.211 \\
\hline Single-port VATS & $12(38 \%)$ & $2(25 \%)$ & 0.689 \\
\hline \multicolumn{4}{|l|}{ Extent of resection } \\
\hline Wedge resection only & $30(94 \%)$ & $8(100 \%)$ & 1.000 \\
\hline Mortality & 0 & 0 & \\
\hline Major morbidity & $1(3 \%)$ & 0 & 1.000 \\
\hline Persistent air leak & $1(3 \%)$ & 0 & 0.564 \\
\hline Chest drain duration (days) & $2.7 \pm 1.7$ & $2.5 \pm 1.5$ & 0.817 \\
\hline Length of stay (days) & $3.5 \pm 2.0$ & $2.9 \pm 1.4$ & 0.450 \\
\hline
\end{tabular}

VATS, none of the pneumothoraxes required additional intervention. There was no clinically significant bleeding from hookwire insertion. There was also no additional time required for interdepartmental transfer. The mean 'at risk' period between the end of hookwire localization and skin incision for VATS was $41.1 \pm 15.0$ minutes. Single-port VATS was performed in $12(38 \%)$ patients. No hookwires were found dislodged leading to failure of localization. 
Table 3 Histopathology of the resected lung nodules from iVATS vs. sVATS

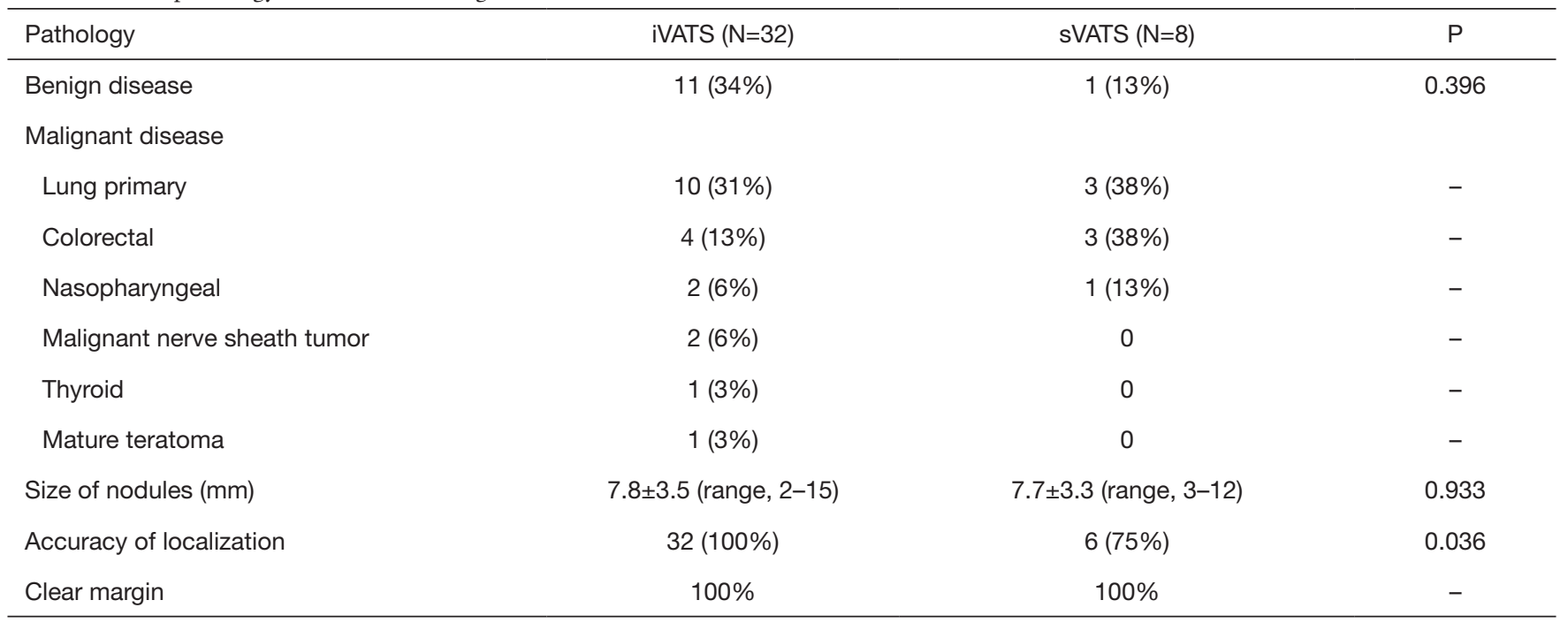

Patients recovered uneventfully after the operation. The post-operative outcomes are summarized in Table 2. There were no mortality or major morbidities. The mean chest drain duration was $2.7 \pm 1.7$ days, and the mean length of hospital stay was $3.5 \pm 2.0$ days.

Eleven patients had benign diseases (such as tuberculosis, organizing pneumonia, and fibrosis) (Table 3). Malignant diseases were diagnosed in the remaining $66 \%$ patients. Primary lung cancer was the commonest detected malignancy $(\mathrm{N}=10,31 \%)$, followed by metastatic colorectal cancer, nasopharyngeal cancer, malignant peripheral nerve sheath tumor, papillary thyroid carcinoma, and mature teratoma. Mean size of the pulmonary nodule on pathological examination was $7.8 \pm 3.5 \mathrm{~mm}$ (range, $2-15 \mathrm{~mm}$ ). Accurate localization was achieved for all the lung nodules. On pathological examination, all the resection margins were clear of tumor involvement.

In comparison, eight patients received hookwire insertion followed by VATS wedge resection in separate procedural and operation suites (sVATS) before the hybrid theatre was available. The technical aspects of the sVATS cohort were compared with the iVATS cohort. The mean size, depth and consistency of the lung nodules in sVATS were comparable with those who received iVATS. Those who had hookwire insertion in the radiology suits have significantly longer 'at risk' period $(109.5 \pm 57.1$ minutes for sVATS $v s .41 .1 \pm 15.0$ minutes for iVATS, $\mathrm{P}=0.011)$. In sVATS group, six out of eight patients developed pneumothorax (75\% for sVATS vs. $47 \%$ for iVATS, $\mathrm{P}=0.154$ ) after hookwire insertion though none of them required additional intervention, and hookwire dislodgement happened in two patients (25\% for sVATS vs. 0 for iVATS, $\mathrm{P}=0.036$ ), resulting in the lung nodules requiring manual palpation for localization.

\section{Discussion}

Localization is an important step in successful resection of small pulmonary nodules. Digital palpation is the simplest way of intraoperative localization; however, for small nodules, soft or ill-defined in consistency, or distant from the lung surface, localization by digital palpation during minimally-invasive approaches can be very challenging and unreliable. Surgeons have lack of tactile feedback when operating with long instruments through small ports, adding further difficulty to proper localization. Advancing from the traditional 3-port VATS, nowadays single-incision, or single port VATS, which utilize only one $2.5-3.5 \mathrm{~cm}$ intercostal incision, has become a popular and rapidly developing ultra-minimally invasive approach applicable to even major lung resections (4-6). However, finger palpation whilst sharing a small single incision with the fencing instruments adds difficulties to digital localization of lung nodules. Various adjuncts, such as hookwire, dye injection, endoscopic ultrasound, percutaneous microcoils and others have been described to help localization (7). Hookwire insertion under computed tomography (CT) guidance is the commonest modalities of localization of lung nodules prior to wedge resection. It is well recognized and reported to have high accuracy of localization and resection (success rate: $92.3-100 \%)(8-14)$. However, hookwire insertion 
is not without risks. The incidence of pneumothorax was $18.6-40 \%(8-10,12)$, and in one series $2.1 \%$ patients were complicated with pneumothorax requiring chest drain insertion (13). Our pneumothorax rate is high as we reported regardless of size all pneumothorax seen on dyna/CT scan. Hemorrhage from needle puncture happened in $5.9-36 \%(8-10,12,13)$. Risk of hookwire migration or dislodgement was $2.4-3.7 \%(12,13)$. Moreover, there is a very small risk of air embolism from hookwire puncture. The procedural risks are unavoidable, but the progression of such and the need of additional intervention before the lung surgery can be minimized if the time between localization procedure and the operation can be minimized, by performing the two treatments in the same location. Reducing patient transfer between the radiology suite, the general ward, and the operating theatre can also reduce the chance of excessive body motions and the subsequent chance of hookwire migration.

The hybrid OR setting allows a one-stop operation, and therefore can well-address the above issues. In our iVATS workflow, VATS lung wedge resection immediately follows the hookwire insertion, therefore there is almost no chance for significant progression of pneumothorax or bleeding complications (which are significant safety concerns for patients with chronic obstructive pulmonary disease or bleeding diathesis). No additional patient transfer is required, so the risk of hookwire migration or dislodgement is minimized. The accuracy of hookwire position can be reconfirmed just prior to wedge resection with the help of real-time CT imaging inside the hybrid OR. All in all, iVATS provided additional advantages in terms of procedural safety and localization accuracy over the traditional method. The hybrid OR is also a conflux of multidisciplinary team work between the interventional radiologists, anesthesiologists and thoracic surgeons. A successful iVATS requires discussion and planning between the 3 specialties prior to the operation.

Other consideration when implementing the iVATS program is the cost, which would be highly variable depending on regional healthcare costs, the hybrid system installed (approximately 3 million USD), the case number/ utilization of the hybrid OR, and other variables such as local system maintenance costs and amortization. Although the hybrid OR requires an initial set-up cost, it could also bring cost benefits due to fewer patient transfer, less porter requirement, and time-saving, and potentially cuts off the costs from repeated imaging and follow-up.
The hookwire insertion in the hybrid OR can be performed under local or general anaesthesia. There are advantages of performing the hookwire procedure under general anaesthesia (GA), for example, less patient discomfort during hookwire insertion, and shorter hookwire insertion to surgical incision time. On the other hand, we choose to perform ours under local anaesthesia for several reasons. Firstly, it is easier to ask the patient to cooperate with breath-hold during the procedure or be positioned prone. We experienced patients developing atelectasis under GA making dyna-CT image of tiny GGO lesions difficult to see within the collapsed lung. Lastly, there is theoretical increase risk of pneumothorax developing and air embolism during hookwire insertion with positive pressure ventilation.

We did not report the localization time because the hookwire localization procedure in the radiology department or in hybrid OR were actually done by the same radiologist and technique using CT guidance, hence there is no difference between the study group and control group. The global OR time (from enter the OR to leaving the OR) will be longer for the iVATS group because of the additional procedure of hookwire localization, but of course this is in lieu of the time needed to do the procedure in the radiology department. We feel the "at risk period" (i.e., the delay between finishing hookwire placement and commencement of VATS) more reflects the advantages of the hybrid theatre, amongst them are reducing the risk of pneumothorax progression and hookwire dislodgement.

The current study is limited by its retrospective nature and the relatively small sample size. Nevertheless, it is shown in the comparison that the iVATS workflow provides a significantly shorter "at risk" period, which logically can provide the advantages found in this study, not to mention shorter duration of patient discomfort from the protruding hookwire.

\section{Conclusions}

Our initial experience with the image-guided VATS lung wedge resection for tiny lung nodules in the hybrid operating theatre demonstrated that it is a safe and effective facilitator of wedge resection of tiny pulmonary nodules in selected patients, and may have added advantages in terms of safety and localization accuracy over the traditional method. Although it's clinical benefits and cost-effectiveness need long-term follow-up and further studies, our early results are promising. 


\section{Acknowledgements}

Funding: The study was funded by Earmarked Research Grant, Research Grants Council (RGC), Hong Kong. Ref. No. 14117715 .

\section{Footnote}

Conflicts of Interest: Calvin S. H. Ng and Simon C. H. Yu. have had research collaborations with Siemens, Germany. The other authors have no conflicts of interest to declare.

Ethical Statement: The ethical approval is by the Joint Chinese University of Hong Kong-New Territories East Cluster Clinical Research Ethics Committee (CREC Ref No. 2017-292). Patient consent for the study is not necessary in view of its retrospective nature.

\section{References}

1. Suzuki K, Nagai K, Yoshida J, et al. Video-assisted thoracoscopic surgery for small indeterminate pulmonary nodules: indications for preoperative marking. Chest 1999; 115:563-8.

2. Ng CS, Man Chu C, Kwok MW, et al. Hybrid DynaCT scan-guided localization single-port lobectomy. [corrected]. Chest 2015;147:e76-8.

3. Zhao ZR, Lau RW, Ng CS. Hybrid theatre and alternative localization techniques in conventional and singleport video-assisted thoracoscopic surgery. $\mathrm{J}$ Thorac Dis 2016;8:S319-27.

4. Ng CS, Rocco G, Wong RH, et al. Uniportal and singleincision video-assisted thoracic surgery: the state of the art. Interact Cardiovasc Thorac Surg 2014;19:661-6.

5. Yu PS, Capili F, Ng CS. Single port VATS: recent developments in Asia. J Thorac Dis 2016;8:S302-7.

Cite this article as: $\mathrm{Hu}$ PS, Chu CM, Lau RW, Wan IY, Underwood MJ, Yu SC, Ng CS. Video-assisted thoracic surgery for tiny pulmonary nodules with real-time image guidance in the hybrid theatre: the initial experience. J Thorac Dis 2018;10(5):2933-2939. doi: 10.21037/jtd.2018.05.53
6. Ng CS, Kim HK, Wong RH, et al. Single-Port VideoAssisted Thoracoscopic Major Lung Resections: Experience with 150 Consecutive Cases. Thorac Cardiovasc Surg 2016;64:348-53.

7. Zaman M, Bilal H, Woo CY, et al. In patients undergoing video-assisted thoracoscopic surgery excision, what is the best way to locate a subcentimetre solitary pulmonary nodule in order to achieve successful excision? Interact Cardiovasc Thorac Surg 2012;15:266-72.

8. Chen YR, Yeow KM, Lee JY, et al. CT-guided hook wire localization of subpleural lung lesions for video-assisted thoracoscopic surgery (VATS). J Formos Med Assoc 2007;106:911-8.

9. Seo JM, Lee HY, Kim HK, et al. Factors determining successful computed tomography-guided localization of lung nodules. J Thorac Cardiovasc Surg 2012;143:809-14.

10. Kastl S, Langwieler TE, Krupski-Berdien G, et al. Percutaneous localization of pulmonary nodules prior to thoracoscopic surgery by CT-guided hook-wire. Anticancer Res 2006;26:3123-6.

11. Paci M, Annessi V, Giovanardi F, et al. Preoperative localization of indeterminate pulmonary nodules before videothoracoscopic resection. Surg Endosc 2002;16:509-11.

12. Dendo S, Kanazawa S, Ando A, et al. Preoperative localization of small pulmonary lesions with a short hook wire and suture system: experience with 168 procedures. Radiology 2002;225:511-8.

13. Hanauer M, Perentes JY, Krueger T, et al. Pre-operative localization of solitary pulmonary nodules with computed tomography-guided hook wire: report of 181 patients. J Cardiothorac Surg 2016;11:5.

14. Park CH, Han K, Hur J, et al. Comparative effectiveness and safety of preoperative lung localization for pulmonary nodules: a systematic review and meta-analysis. Chest 2017;151:316-28. 